OPEN ACCESS

Edited by:

$\mathrm{Li} F$,

Hangzhou Dianzi University, China

Reviewed by:

Hassan Karimi-maleh,

University of Electronic Science and

Technology of China, China

Yuhong Zheng,

Jiangsu Province and Chinese

Academy of Sciences, China

*Correspondence:

Jinying Zhang

Jinyingzhang2021@163.com

Yiqiang Yuan

yqyuan@sutcm.net

Specialty section:

This article was submitted to

Electrochemistry,

a section of the journal

Frontiers in Chemistry

Received: 15 March 2021 Accepted: 26 April 2021

Published: 14 May 2021

Citation:

Li J, Zhang S, Zhang L, Zhang Y, Zhang $H$, Zhang $C$, Xuan $X$, Wang $M$, Zhang $J$ and Yuan $Y$ (2021) A Novel

Graphene-Based Nanomaterial Modified Electrochemical Sensor for the Detection of Cardiac Troponin I.

Front. Chem. 9:680593.

doi: 10.3389/fchem.2021.680593

\section{A Novel Graphene-Based Nanomaterial Modified Electrochemical Sensor for the Detection of Cardiac Troponin I}

\author{
Jing Li ${ }^{1}$, Shenwei Zhang ${ }^{1}$, Li Zhang ${ }^{2}$, Yu Zhang ${ }^{1}$, Hua Zhang ${ }^{1}$, Chuanxi Zhang ${ }^{1}$, Xuexi Xuan ${ }^{1}$, \\ Mingjie Wang ${ }^{1}$, Jinying Zhang ${ }^{2 *}$ and Yiqiang Yuan ${ }^{3 *}$
}

${ }^{1}$ Department of Cardiology, The Seventh People's Hospital of Zhengzhou, Zhengzhou, China, ${ }^{2}$ Department of Cardiology, The First Affiliated Hospital of Zhengzhou University, Zhengzhou, China, ${ }^{3}$ Department of Cardiology, Chest Hospital of Henan Provincial, Zhengzhou, China

Acute myocardial infarction has a high clinical mortality rate. The initial exclusion or diagnosis is important for the timely treatment of patients with acute myocardial infarction. As a marker, cardiac troponin I (cTnl) has a high specificity, high sensitivity to myocardial injury and a long diagnostic window. Therefore, its diagnostic value is better than previous markers of myocardial injury. In this work, we propose a novel aptamer electrochemical sensor. This sensor consists of silver nanoparticles/MoS $/$ reduced graphene oxide. The combination of these three materials can provide a synergistic effect for the stable immobilization of aptamer. Our proposed aptamer electrochemical sensor can detect cTnl with high sensitivity. After optimizing the parameters, the sensor can provide linear detection of cTnl in the range of $0.3 \mathrm{pg} / \mathrm{ml}$ to $0.2 \mathrm{ng} / \mathrm{ml}$. In addition, the sensor is resistant to multiple interferents including urea, glucose, myoglobin, dopamine and hemoglobin.

Keywords: aptamer electrochemical sensor, reduced graphene oxide, troponin I, mos2, glassy carbon electrode

\section{INTRODUCTION}

Troponin is a regulatory heteropeptide protein found in myogenic fiber filaments that plays a pivotal role in the interaction between actin and myosin. It controls contraction and relaxation of skeletal and cardiac muscles. Troponin is a complex that includes three subunits: ion-binding troponin $(\mathrm{cTnC})$, troponin that inhibits actin-myosin interaction $(\mathrm{cTnI})$ and tropomyosin $(\mathrm{cTnT})$, which is used to bind the myosin complex to promyosin and promote myocardial contraction (Fan et al., 2018; Miao et al., 2019; Phonklam et al., 2020). It has been demonstrated that cTnI is a specific biomarker for myocardial injury in dogs, cats, horses, pigs, goats, mice and cattle. Damage to myocardial cell integrity is followed by partial release of cTnI into the blood, and elevated cardiac troponin concentrations in peripheral blood indicate myocardial cell damage (Palladino et al., 2018; Karimi-Maleh et al., 2020a; Çimen et al., 2020). Since myocardial infarction is one of the most important factors leading to myocardial cell destruction, monitoring of cTnI blood concentrations is particularly important for the early detection of myocardial infarction (Ye et al., 2018; Lee et al., 2019; Sun et al., 2019a; Karimi-Maleh et al., 2020b, 2021). There is evidence that even small elevations in cTnI may be associated with poor prognosis. However, as with most other current assays, their most significant shortcoming lies within the first few hours of acute myocardial infarction onset. Current cTnI assays are not effective in detecting elevated blood concentrations and are not effective in 
monitoring changes in lower concentrations of cTnI. Therefore, the development and application of next-generation cTnI assays is imminent (Negahdary et al., 2017; Zhang et al., 2018; Fu et al., 2019b; Sun et al., 2019b; Zhou et al., 2020).

Radioimmunoassay is a method by which an isotope-labeled antigen is added to an unlabeled antigen to cause a competitive inhibition reaction with the antibody. The radioimmunoassay is equal to the advantages due to its good utility and specificity, as well as its high sensitivity. However, the method has disadvantages such as the existence of radioactive contamination and short half-life of isotopes, which to a certain extent limit the development and application of radioimmunoassay (Qiao et al., 2018; Sarangadharan et al., 2018; Fu et al., 2019a; Wang et al., 2019; Mokhtari et al., 2020; Xu et al., 2020). Fluorescent immunoassay is mainly used for the diagnosis of infectious diseases due to its high sensitivity, and it is one of the oldest labeled immunoassays. However, the detection limit of fluorescence immunoassay is not low enough. Moreover, it is difficult to store fluorescent samples for a long time. Electrochemical immunosensor is an application that combines electrochemiluminescence measurement with immunosensor. It combines the advantages of high sensitivity of electrochemiluminescence and high selectivity of immunoassay, which has attracted the attention of many researchers (Dhawan et al., 2018; Regan et al., 2018; Karimi-Maleh et al., 2020c; Wang et al., 2020). The unique physicochemical properties of nanomaterials make it a promising application in the development of high-performance electrochemical and electrochemiluminescent sensors.

Two-dimensional nano has been widely studied for its high specific surface area and compatibility with miniaturized devices (Khodadadi et al., 2019; Tahernejad-Javazmi et al., 2019). Among them, $\mathrm{MoS}_{2}$, in which Mo atomic layers are arranged in a hexagonal shape between $S$ atomic layers, has an ultrathin planar structure. It is sensitive to the surrounding environment and becomes a suitable base material for building aptamer sensors (Cai et al., 2018; Chekin et al., 2018). However, $\mathrm{MoS}_{2}$ has low electrical conductivity and Van Der Waals between the layers tend to agglomerate it. Reduced graphene (rGO) with active edge sites and excellent electrical properties can effectively improve the electrochemical activity of $\mathrm{MoS}_{2}$. The composite of other nanomaterials in $\mathrm{rGO}$ can reduce the layer-layer interaction force, so the performance of rGO can be further ensured (Fathil et al., 2017; Lopa et al., 2019). Among them, silver nanomaterial materials are often used in the design of electrochemical sensors due to their cheap price and good electrocatalytic properties (Zhou et al., 2018; Yan et al., 2019).

This work synthesized AgNPs/ $\mathrm{MoS}_{2} / \mathrm{rGO}$ nanocomposites. By combining the excellent properties of the composite (large surface area and good electrical conductivity) with the aptamer (high affinity and specificity), a label-free electrochemical aptamer sensor was developed for the sensitive and selective detection of cTnI.

\section{MATERIALS AND METHODS}

cTnI and DNA oligonucleotides were obtained from Yeyuan Biotech. Urea, thrombin, myoglobin, horseradish peroxidase,
L-cysteine, hemoglobin, and prostate-specific antigen were ordered from Alading Co. Ltd. Graphene oxide (GO) powder was purchased from Xianfeng Nano Tech Co. Ltd. All other reagents are analytically pure and can be used without additional purification. $0.1 \mathrm{M}$ Tris- $\mathrm{HCl}$ buffer solution has been used for dissolving amino-modified cTnI aptamer (AcTnl).

Sequence of the AcTnl is: $5^{\prime}-\mathrm{NH}_{2}-\mathrm{C}_{6} \mathrm{H}_{12}$-CGTGCAGTAC GCCAACCTTTCTCATGCGCGCTGCCCCTCTTA- $3^{\prime}$.

All electrochemical tests, including cyclic voltammetry (CV), differential pulse voltammetry (DPV), and electrochemical impedance spectroscopy (EIS) were performed using the CHI660 electrochemical analyzer/workstation with threeelectrode system. SEM image has been recorded using a ZEISS MERLIN.

Synthesis of AgNPs/ $\mathrm{MoS}_{2} / \mathrm{rGO}$ nanocomposite: $50 \mathrm{mg}$ of $\mathrm{AgNO}_{3}$ was dissolved in $20 \mathrm{ml}$ of ethanol and $100 \mathrm{mg}$ of octadecylamine was added with vigorous stirring. After complete dissolution, $50 \mathrm{mg}$ of glucose and $60 \mathrm{mg}$ of glycine were added and sonicated. The solution was transferred to a hydrothermal kettle and heated at $120^{\circ} \mathrm{C}$ for overnight. Silver nanoparticles (AgNPs) were obtained after filtration. $\mathrm{MoS}_{2} / \mathrm{rGO}$ was prepared by adding $10 \mathrm{mg} \quad \mathrm{Na}_{2} \mathrm{MoO}_{4} \cdot 2 \mathrm{H}_{2} \mathrm{O}$ and $20 \mathrm{mg}$ L-cysteine into $20 \mathrm{ml}$ of $\mathrm{GO}$ dispersion $(0.5 \mathrm{mg} / \mathrm{ml}$, $\mathrm{DMF}$ ). The mixture was then transferred into a hydrothermal kettle and heated at $100^{\circ} \mathrm{C}$ for $5 \mathrm{~h}$. The $\mathrm{MoS}_{2} / \mathrm{rGO}$ was collected after the filtration. The AgNPs/ $\mathrm{MoS}_{2} / \mathrm{rGO}$ nanocomposite was prepared by adding AgNPs into $\mathrm{MoS}_{2} / \mathrm{rGO}$ composite dispersion after $1 \mathrm{~h}$ sonication.

Electrode fabrication: A glassy carbon electrode (GCE) has been used for sensor fabrication. Specifically, a certain amount of AgNPs/ $\mathrm{MoS}_{2} / \mathrm{rGO}$ nanocomposite was drop casted on a GCE and dried naturally. Then, a certain amount of AcTnl was drop casted on the AgNPs/MoS $2 / \mathrm{rGO} / \mathrm{GCE}$. After drying, the AcTnl/ $\mathrm{AgNPs} / \mathrm{MoS}_{2} / \mathrm{rGO}$ was incubated in the cTnl solution with different concentration for $1 \mathrm{~h}$ before electrochemical signal recording.

\section{RESULTS AND DISCUSSION}

Figure 1 demonstrates the preparation and sensing strategy of this label-free electrochemical aptamer sensor. $\mathrm{AgNPs} / \mathrm{MoS}_{2} /$ rGO immobilized with GCE can immobilize the AcTnl aptamer on the electrode surface via $\mathrm{Ag}-\mathrm{N}$ bond. In this process, the electrochemical signal of $\left[\mathrm{Fe}(\mathrm{CN})_{6}\right]^{3-/ 4-}$ decreases because the electron transfer is hindered by the nucleobases. When AcTnl/AgNPs/ $\mathrm{MoS}_{2} / \mathrm{rGO}$ is immersed in the cTnI solution, the electrochemical signal is further reduced due to the formation of AcTnI-cTnI complexes on the electrode surface that further hinder electron transfer. By calculating the relationship between peak current and cTnI concentration, the detection of cTnI concentration can be achieved.

Figure 2 shows the SEM images of AgNPs, $\mathrm{MoS}_{2} / \mathrm{rGO}$, and $\mathrm{AgNPs} / \mathrm{MoS}_{2} / \mathrm{rGO}$. The structure of silver nanoflowers can be clearly observed in Figure 2A. The SEM images of $\mathrm{MoS}_{2} / \mathrm{rGO}$ show the typical wrinkled and folded structure of graphene and the layer-like structure of $\mathrm{MoS}_{2} / \mathrm{rGO}$. The tight contact between 


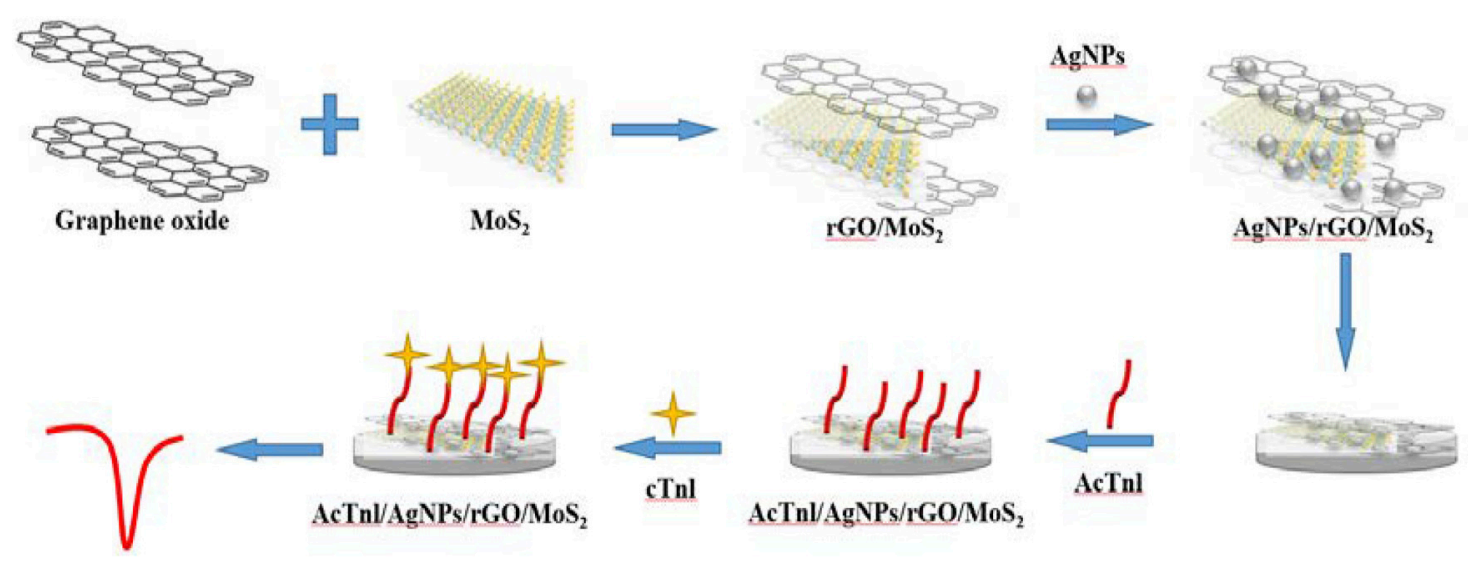

FIGURE 1 | Schematic diagram for the preparation of the AcTnl/AgNPs/MoS $/$ /rGO sensor for cTnl detection.
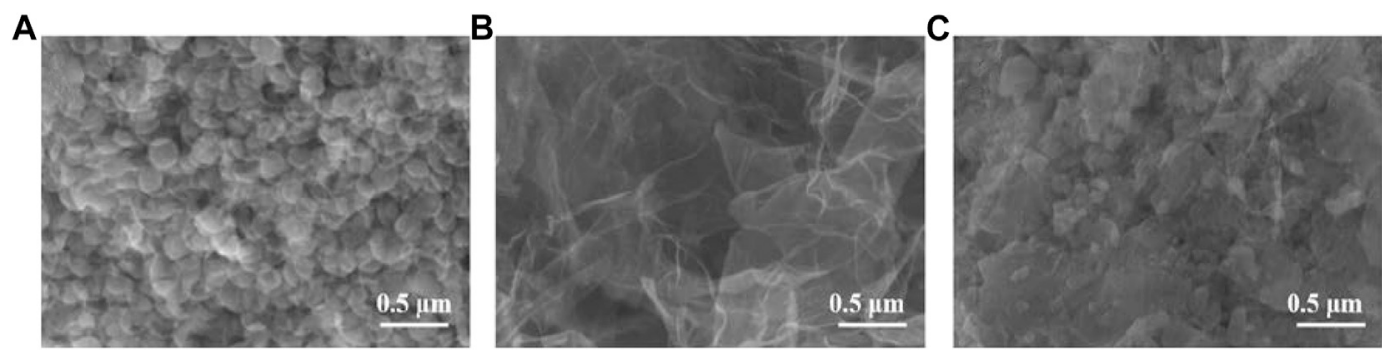

FIGURE 2 | SEM images of (A) AgNPs (B) $\mathrm{MoS}_{2} / \mathrm{rGO}$, and (C) AgNPs/MoS $2 / \mathrm{rGO}$.

$\mathrm{MoS}_{2}$ and rGO is favorable for electron transfer. The SEM images of AgNPs $/ \mathrm{MoS}_{2} / \mathrm{rGO}$ nanocomposites clearly observe that AgNPs are wrapped by $\mathrm{MoS}_{2} / \mathrm{rGO}$ composites.

We tested the electrochemical behavior of the electrode surface during sensor assembly using a $5.0 \mathrm{mM}\left[\mathrm{Fe}(\mathrm{CN})_{6}\right]^{3-14-}$ solution containing $0.1 \mathrm{M} \mathrm{KCl}$ (Figure 3). The redox peak current of the modified GCE was significantly increased due to the excellent conductivity and electron transfer of rGO. After the modification of GCE with $\mathrm{MoS}_{2} / \mathrm{rGO}$ and AgNPs/MoS$/ \mathrm{rGO}$, the currents increased significantly in turn, which was caused by the large specific surface area of $\mathrm{MoS}_{2}$ and the excellent electrochemical properties of AgNPs.

The electrochemical properties of the sensor was further investigated by EIS. In EIS, semicircular and straight regions indicate electron transfer-limited processes and mass transfer control processes (Demirbakan and Kemal Sezgintürk, 2020; Lee et al., 2020), respectively. Considering the variation of the electron transfer resistance $\left(\mathrm{R}_{\mathrm{ct}}\right)$, the EIS method can provide important information to demonstrate the interaction between the aptamer and the target protein. As shown in Figure 4, bare GCE presents a small semicircle with an $\mathrm{R}_{\mathrm{ct}}$ value of $266 \Omega$. Due to the excellent properties and synergistic effects of rGO, $\mathrm{MoS}_{2}$ and AgNPs, the $\mathrm{R}_{\mathrm{ct}}$ value decreases to $91 \Omega$ after AgNPs/MoS $2 /$ rGO modification, indicating that the electron transfer effect is enhanced. This is consistent with the CV results of GCE and $\mathrm{AgNPs} / \mathrm{MoS}_{2} / \mathrm{rGO} / \mathrm{GCE}$. While the presence of AcTnI leads to an increase in the spatial site blocking effect and hinders electron transfer, the $\mathrm{R}_{\mathrm{ct}}$ increases sharply to occur at 3.31 $\mathrm{k} \Omega$, indicating the binding of AcTnI and AgNWs on the electrode surface. With the binding of the target protein on the AcTnI/AgNPs $/ \mathrm{MoS}_{2} / \mathrm{rGO} / \mathrm{GCE}$ surface, a continued increase in $\mathrm{R}_{\mathrm{ct}}$ to $5.26 \mathrm{k} \Omega$ can be observed, indicating a successful binding with the target protein to its aptamer, which hinders the electron transfer.

In order to improve the sensitivity of detecting cTnI, different parameters were optimized. Figure $\mathbf{5 A}$ shows the aptamer electrodes assembled with different concentrations of AgNPs in the $\mathrm{MoS}_{2} / \mathrm{rGO}$ dispersion. Although AgNPs have excellent electrochemical properties, when AgNPs exceed a certain concentration it may cause a high stacking density, resulting in a lower detection efficiency of the sensor. Therefore, we chose to add $250 \mu$ Lof AgNPs into the $\mathrm{MoS}_{2} / \mathrm{rGO}$ dispersion.

The amount of modification of $\mathrm{AgNPs} / \mathrm{MoS}_{2} / \mathrm{rGO}$ is an important factor affecting the immobilization of AcTnI. Figure 5B shows the electrochemical response of the proposed aptamer sensor $\mathrm{AgNPs} / \mathrm{MoS}_{2} / \mathrm{rGO}$ at different modification amounts. As the concentration of $\mathrm{AgNPs} / \mathrm{MoS}_{2} / \mathrm{rGO}$ increases, the electron transfer resistance of the interface may increase, 


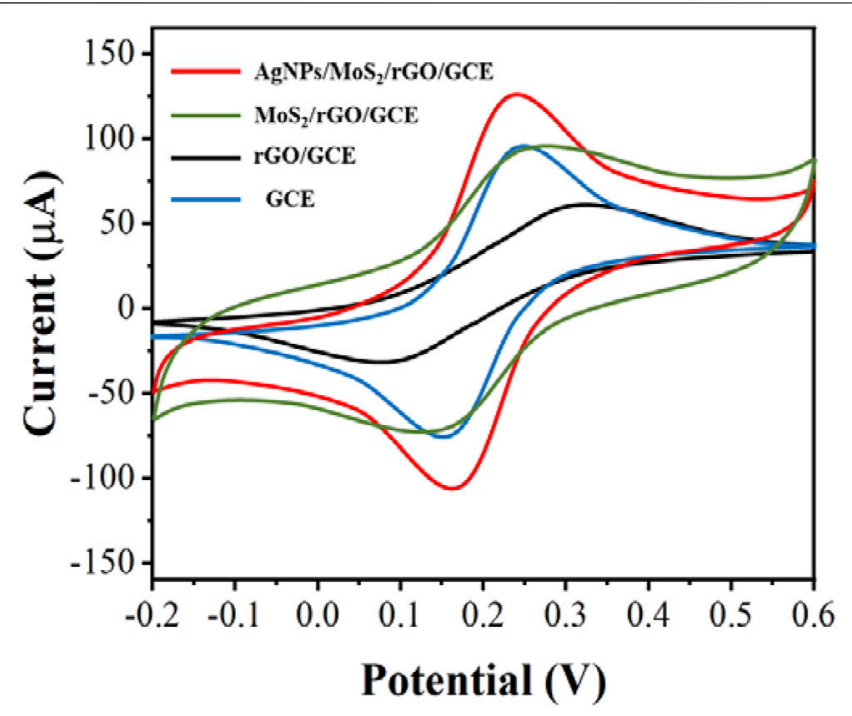

FIGURE 3 | CV of GCE, rGO/GCE, MoS $2 / \mathrm{rGO} / \mathrm{GCE}$ and AgNPs/MoS $2 / \mathrm{rGO} / \mathrm{GCE}$ in $5.0 \mathrm{mM}\left[\mathrm{Fe}(\mathrm{CN})_{6}\right]^{3-/ 4-}$. Scan rate: $50 \mathrm{mV} / \mathrm{s}$.

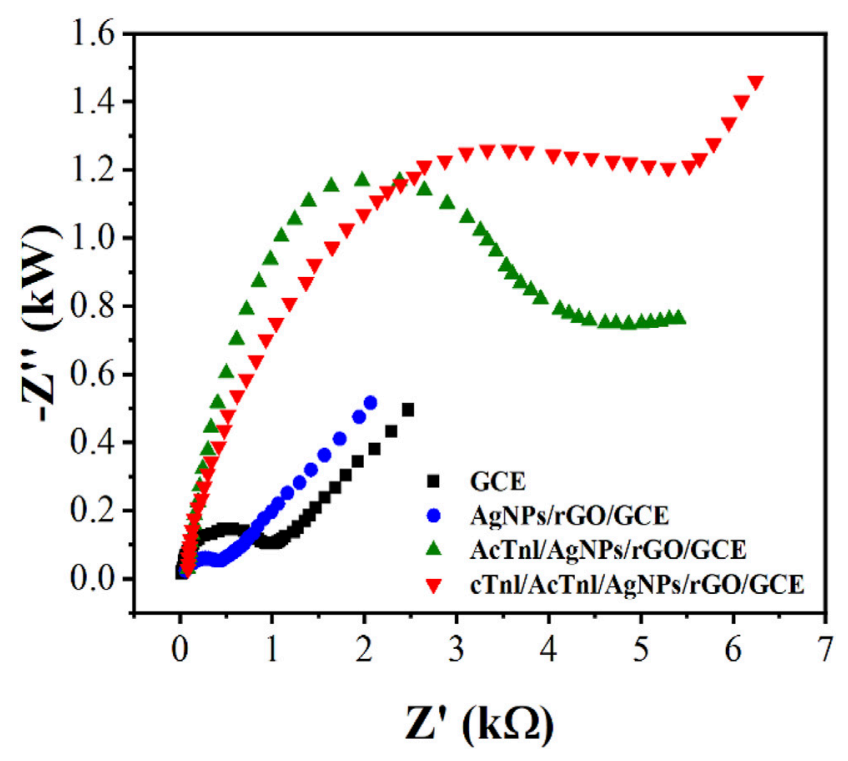

FIGURE 4 | EIS of GCE, rGO/GCE, $\mathrm{MoS}_{2} / \mathrm{rGO} / \mathrm{GCE}$ and $\mathrm{AgNps} / \mathrm{MoS}_{2} / \mathrm{rGO} / \mathrm{GCE}$ in $5.0 \mathrm{mM}\left[\mathrm{Fe}(\mathrm{CN})_{6}\right]^{3-/ 4-}$.

while there may be some interference with the detection of cTnI. Therefore, $5 \mu \mathrm{L}$ of $\mathrm{AgNPs} / \mathrm{MoS}_{2} / \mathrm{rGO}$ dispersion was obtained as the optimal condition.

The concentration of AcTnI affects the charge transfer efficiency of the electrode interface for detecting cTnI. Figure $6 \mathbf{A}$ shows the effect of different AcTnI concentrations on the DPV signal of AgNPs/MoS $2 /$ rGO. When the concentration reaches $3 \mu \mathrm{M}$, the DPV response is significantly reduced, indicating that the surface active site of the sensor may be fully occupied. Considering the size of the aptamer itself, the tightly packed surface prevents the aptamer from specifically binding cTnI. because we chose $3 \mu \mathrm{M}$ AcTnI as the optimal concentration for the aptamer sensor.

The incubation time of cTnI is also another important factor affecting cTnI detection. As shown in Figure 6B, the electrochemical signal did not change significantly after the incubation time of AcTnI reached $30 \mathrm{~min}$. This indicates that the bioaffinity between AcTnI on the electrode surface and the cTnI target saturated. On the other hand, the current response stabilizes due to the spatial potential resistance effect, leading to a decrease in sensitivity to the target. Therefore, we chose $30 \mathrm{~min}$ as the optimal incubation time. 

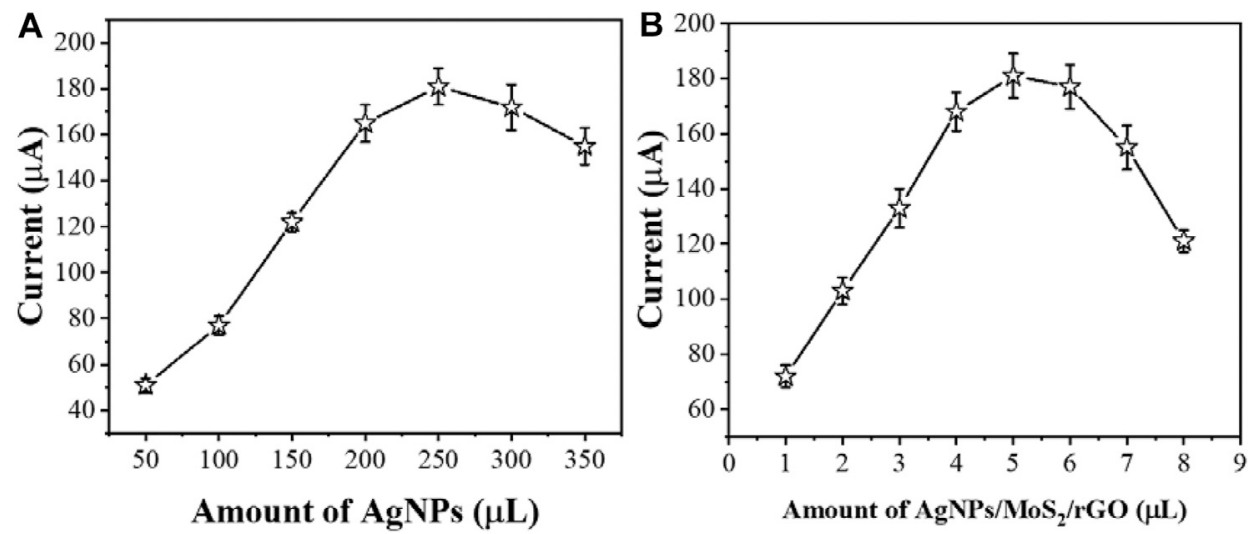

FIGURE 5 | The effect of (A) amount of AgNPs and (B) AgNPs/MoS $2 /$ rGO composite for sensing performance $(n \geq 3)$.
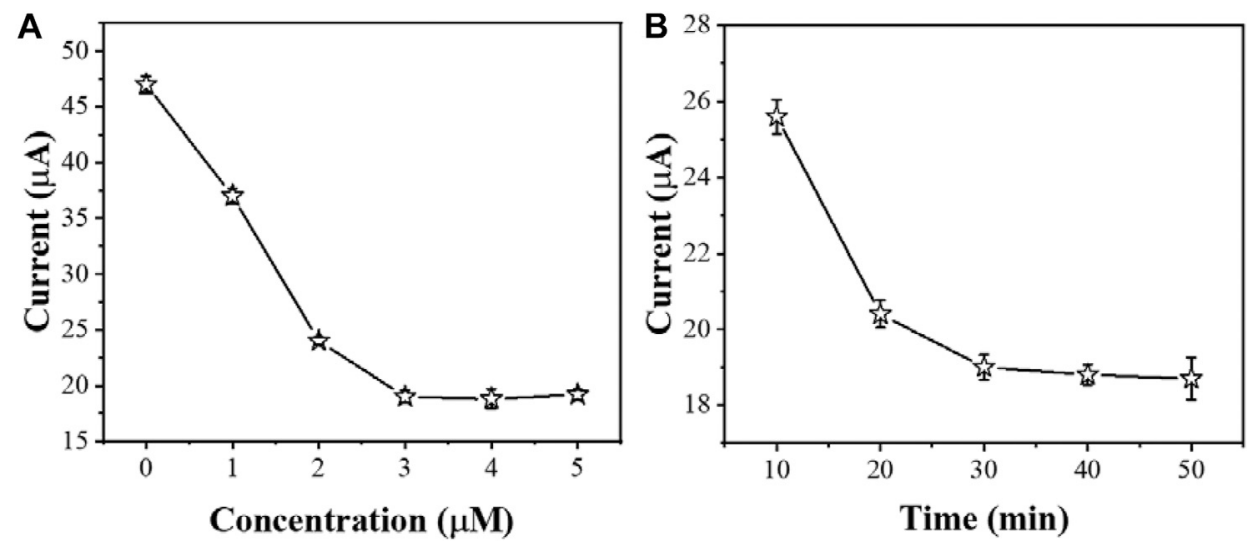

FIGURE 6 | The effect of $(\mathbf{A})$ amount of AcTnl and $\mathbf{( B )}$ incubation time for sensing performance $(n \geq 3)$.
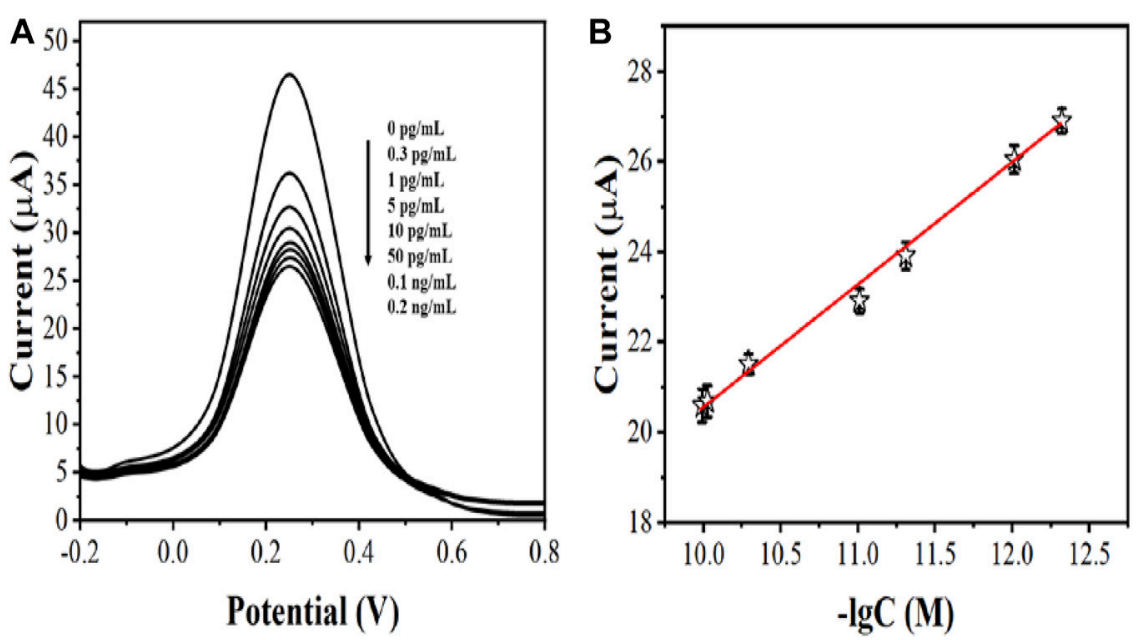

FIGURE 7 | (A) DPV curves of AcTnl/AgNPs/MoS $2 / \mathrm{rGO} / \mathrm{GCE}$ toward 0 pg/ml, $0.3 \mathrm{pg} / \mathrm{ml}, 1 \mathrm{pg} / \mathrm{ml}, 5 \mathrm{pg} / \mathrm{ml}, 10$ pg/ml, 50 pg/ml, $0.1 \mathrm{ng} / \mathrm{ml}$, and $0.2 \mathrm{ng} / \mathrm{ml}$ (B) Linear relationship between current and logarithm of the cTnl concentrations $(n \geq 3)$. 


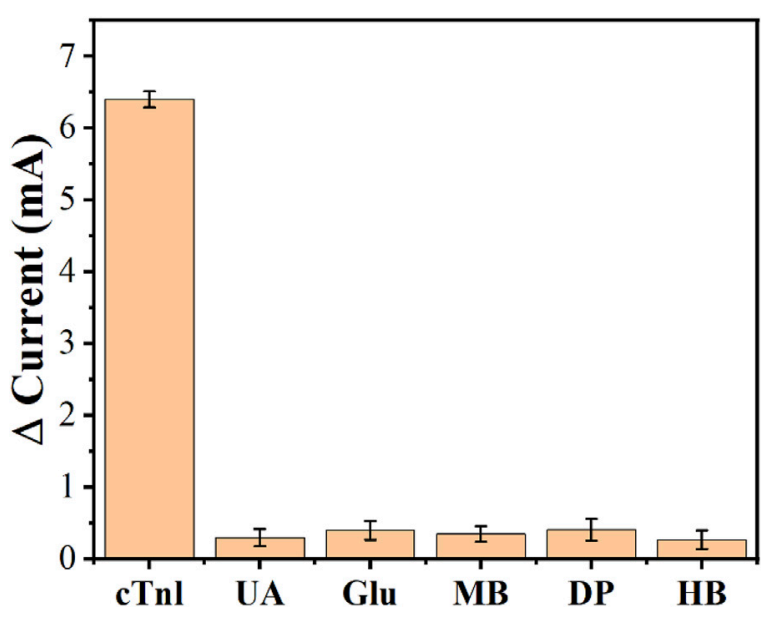

FIGURE 8 | Anti-interference performance of the AcTnl/AgNPs/MoS $2 / r G O / G C E$ toward UA, Glu, MB, DP, and HB.

After optimizing the parameters, we immersed AcTnI/AgNPs/ $\mathrm{MoS}_{2} / \mathrm{rGO} / \mathrm{GCE}$ in different concentrations of cTnI solutions. Then, we detected the electrochemical signal by DPV in a $5 \mathrm{mM}$ $\left[\mathrm{Fe}(\mathrm{CN})_{6}\right]^{3-/ 4-}$ solution containing $0.5 \mathrm{M} \mathrm{KCl}$ (Figure 7A). We recorded the linear relationship between the peak value of DPV and the negative logarithm of the concentration of the cTnI standard solution to establish a standard working curve (Figure 7B). It can be seen that the DPV signal correlates well with the negative logarithm of the cTnI concentration in the range of $0.3 \mathrm{pg} / \mathrm{mL}-0.2 \mathrm{ng} / \mathrm{ml}$ with the linear equation: $\operatorname{Ip}(\mu \mathrm{A})=$ $-2.73 \log \mathrm{c}(\mathrm{g} / \mathrm{ml})-6.41\left(R^{2}=0.9947\right)$ and the limit of detection limit was $0.27 \mathrm{pg} / \mathrm{ml}$.

To examine the performance of the aptamer sensor, we tested the reproducibility, stability and specificity of AcTnI/ AgNPs $/ \mathrm{MoS}_{2} / \mathrm{rGO} / \mathrm{GCE}$. The RSD of $1.72 \%$ was recorded by checking 5 parallel measurements on the same sensor, indicating that the electrochemical aptamer sensor for cTnI detection has good reproducibility. The stability of the prepared aptamer sensor was further evaluated by measuring the DPV current of the modified electrode after one month. The electrochemical signal before and after storage changed slightly with a $5.2 \%$ decrease in the peak DPV, demonstrating the good stability of the proposed electrochemical aptamer sensor for cTnI detection.

To determine the selectivity of the developed aptamer sensor, a series of proteins were used for comparison, such as: urea (UA), glucose (Glu), myoglobin (MB), dopamine (DP), and hemoglobin (HB). Among them, the concentration of cTnI was $50 \mathrm{pg} / \mathrm{ml}$ and the concentration of other infectants was $0.2 \mathrm{ng} / \mathrm{ml}$ due to the excellent specific discrimination between cTnI and AcTnI, the aptamer sensor for interferers showed negligible change in current response compared to cTnI, and the results are shown in Figure 8. The proposed aptamer sensor has good selectivity and specific anti-interference ability.

\section{CONCLUSION}

In summary, an aptamer electrochemical sensor was constructed using $\mathrm{AgNPs} / \mathrm{MoS}_{2} / \mathrm{rGO}$ nanocomposite. By combining the excellent properties of the composite (large surface area and good electrical conductivity) with the aptamer (high affinity and specificity), a label-free electrochemical aptamer sensor was developed for the sensitive and selective detection of cTnI. After the optimizations, the proposed aptamer sensor can linear detect cTnl between $0.3 \mathrm{pg} / \mathrm{mL}-0.2 \mathrm{ng} / \mathrm{ml}$ with a low limit of detection of $0.27 \mathrm{pg} / \mathrm{ml}$. In addition, the proposed aptamer sensor has good selectivity and specific anti-interference ability.

\section{DATA AVAILABILITY STATEMENT}

The original contributions presented in the study are included in the article/Supplementary Material, further inquiries can be directed to the corresponding authors.

\section{AUTHOR CONTRIBUTIONS}

JL and SZ conceived of the study. JZ and YY supervised the development program, LZ, JL, and HZ conducted the materials characterization. YZ, CZ, and XX received and curated samples and analytical records. SZ, MW, and JZ wrote the manuscript. All authors read and approved of the manuscript.

\section{FUNDING}

This work was funded by Science and Technology Research Plan Joint Construction Project of Henan Province (LHGJ20191112). 


\section{REFERENCES}

Cai, Y., Kang, K., Li, Q., Wang, Y., and He, X. (2018). Rapid and Sensitive Detection of Cardiac Troponin I for Point-Of-Care Tests Based on Red Fluorescent Microspheres. Molecules 23, 1102. doi:10.3390/molecules23051102

Chekin, F., Vasilescu, A., Jijie, R., Singh, S. K., Kurungot, S., Iancu, M., et al. (2018). Sensitive Electrochemical Detection of Cardiac Troponin I in Serum and Saliva by Nitrogen-Doped Porous Reduced Graphene Oxide Electrode. Sensors Actuators B: Chem. 262, 180-187. doi:10.1016/j.snb. 2018.01.215

Çimen, D., Bereli, N., Günaydın, S., and Denizli, A. (2020). Detection of Cardiac Troponin-I by Optic Biosensors with Immobilized Anti-cardiac Troponin-I Monoclonal Antibody. Talanta 219, 121259. doi:10.1016/j. talanta.2020.121259

Demirbakan, B., and Kemal Sezgintürk, M. (2020). A Novel Ultrasensitive Immunosensor Based on Disposable Graphite Paper Electrodes for Troponin T Detection in Cardiovascular Disease. Talanta 213, 120779. doi:10.1016/j.talanta.2020.120779

Dhawan, S., Sadanandan, S., Haridas, V., Voelcker, N. H., and Prieto-Simón, B. (2018). Novel Peptidylated Surfaces for Interference-free Electrochemical Detection of Cardiac Troponin I. Biosens. Bioelectron. 99, 486-492. doi:10. 1016/j.bios.2017.08.024

Fan, D., Bao, C., Khan, M. S., Wang, C., Zhang, Y., Liu, Q., et al. (2018). A Novel Label-free Photoelectrochemical Sensor Based on N,S-GQDs and CdS Cosensitized Hierarchical $\mathrm{Zn} 2 \mathrm{SnO} 4$ Cube for Detection of Cardiac Troponin I. Biosens. Bioelectron. 106, 14-20. doi:10.1016/j.bios.2018.01.050

Fathil, M. F. M., Md Arshad, M. K., Ruslinda, A. R., Gopinath, S. C. B., Nuzaihan M.N., M., Adzhri, R., et al. (2017). Substrate-gate Coupling in ZnO-FET Biosensor for Cardiac Troponin I Detection. Sensors Actuators B: Chem. 242, 1142-1154. doi:10.1016/j.snb.2016.09.131

Fu, L., Wu, M., Zheng, Y., Zhang, P., Ye, C., Zhang, H., et al. (2019a). Lycoris Species Identification and Infrageneric Relationship Investigation via Graphene Enhanced Electrochemical Fingerprinting of Pollen. Sensors Actuators B: Chem. 298, 126836. doi:10.1016/j.snb.2019.126836

Fu, L., Zheng, Y., Zhang, P., Zhang, H., Wu, M., Zhang, H., et al. (2019b). An Electrochemical Method for Plant Species Determination and Classification Based on Fingerprinting Petal Tissue. Bioelectrochemistry 129, 199-205. doi:10. 1016/j.bioelechem.2019.06.001

Karimi-Maleh, H., Alizadeh, M., Orooji, Y., Karimi, F., Baghayeri, M., Rouhi, J., et al. (2021). Guanine-Based DNA Biosensor Amplified with Pt/SWCNTs Nanocomposite as Analytical Tool for Nanomolar Determination of Daunorubicin as an Anticancer Drug: A Docking/Experimental Investigation. Ind. Eng. Chem. Res. 60, 816-823. doi:10.1021/acs.iecr.0c04698

Karimi-Maleh, H., Ayati, A., Ghanbari, S., Orooji, Y., Tanhaei, B., Karimi, F., et al. (2021c). Recent Advances in Removal Techniques of Cr(VI) Toxic Ion from Aqueous Solution: A Comprehensive Review. J. Mol. Liquids 329, 115062. doi:10.1016/j.molliq.2020.115062

Karimi-Maleh, H., Karimi, F., Malekmohammadi, S., Zakariae, N., Esmaeili, R., Rostamnia, S., et al. (2020a). An Amplified Voltammetric Sensor Based on Platinum Nanoparticle/polyoxometalate/two-Dimensional Hexagonal Boron Nitride Nanosheets Composite and Ionic Liquid for Determination of N-Hydroxysuccinimide in Water Samples. J. Mol. Liquids 310, 113185. doi:10.1016/j.molliq.2020.113185

Karimi-Maleh, H., Kumar, B. G., Rajendran, S., Qin, J., Vadivel, S., Durgalakshmi, D., et al. (2020b). Tuning of Metal Oxides Photocatalytic Performance Using Ag Nanoparticles Integration. J. Mol. Liquids 314, 113588. doi:10.1016/j.molliq. 2020.113588

Khodadadi, A., Faghih-Mirzaei, E., Karimi-Maleh, H., Abbaspourrad, A., Agarwal, S., and Gupta, V. K. (2019). A New Epirubicin Biosensor Based on Amplifying DNA Interactions with Polypyrrole and Nitrogen-Doped Reduced Graphene: Experimental and Docking Theoretical Investigations. Sensors actuators $b$ : Chem. 284, 568-574. doi:10.1016/j.snb.2018.12.164

Lee, K. W., Kim, K. R., Chun, H. J., Jeong, K. Y., Hong, D.-K., Lee, K.-N., et al. (2020). Time-resolved Fluorescence Resonance Energy Transfer-Based Lateral Flow Immunoassay Using a Raspberry-type Europium Particle and a Single Membrane for the Detection of Cardiac Troponin I. Biosens. Bioelectron. 163, 112284. doi:10.1016/j.bios.2020.112284
Lee, T., Ahn, J.-H., Choi, J., Lee, Y., Kim, J.-M., Park, C., et al. (2019). Development of the Troponin Detection System Based on the Nanostructure. Micromachines 10, 203. doi:10.3390/mil0030203

Lopa, N. S., Rahman, M. M., Ahmed, F., Ryu, T., Sutradhar, S. C., Lei, J., et al. (2019). Simple, Low-Cost, Sensitive and Label-free Aptasensor for the Detection of Cardiac Troponin I Based on a Gold Nanoparticles Modified Titanium Foil. Biosens. Bioelectron. 126, 381-388. doi:10.1016/j.bios.2018. 11.012

Miao, L., Jiao, L., Tang, Q., Li, H., Zhang, L., and Wei, Q. (2019). A NanozymeLinked Immunosorbent Assay for Dual-Modal Colorimetric and Ratiometric Fluorescent Detection of Cardiac Troponin I. Sensors Actuators B: Chem. 288, 60-64. doi:10.1016/j.snb.2019.02.111

Mokhtari, Z., Khajehsharifi, H., Hashemnia, S., Solati, Z., Azimpanah, R., and Shahrokhian, S. (2020). Evaluation of Molecular Imprinted Polymerized Methylene Blue/aptamer as a Novel Hybrid Receptor for Cardiac Troponin I (cTnI) Detection at Glassy Carbon Electrodes Modified with New Biosynthesized ZnONPs. Sensors Actuators B: Chem. 320, 128316. doi:10. 1016/j.snb.2020.128316

Negahdary, M., Behjati-Ardakani, M., Sattarahmady, N., Yadegari, H., and Heli, H. (2017). Electrochemical Aptasensing of Human Cardiac Troponin I Based on an Array of Gold Nanodumbbells-Applied to Early Detection of Myocardial Infarction. Sensors Actuators B: Chem. 252, 62-71. doi:10.1016/j.snb.2017. 05.149

Palladino, P., Minunni, M., and Scarano, S. (2018). Cardiac Troponin T Capture and Detection in Real-Time via Epitope-Imprinted Polymer and Optical Biosensing. Biosens. Bioelectron. 106, 93-98. doi:10.1016/j.bios.2018.01.068

Phonklam, K., Wannapob, R., Sriwimol, W., Thavarungkul, P., and Phairatana, T. (2020). A Novel Molecularly Imprinted Polymer PMB/MWCNTs Sensor for Highly-Sensitive Cardiac Troponin T Detection. Sensors Actuators B: Chem. 308, 127630. doi:10.1016/j.snb.2019.127630

Qiao, X., Li, K., Xu, J., Cheng, N., Sheng, Q., Cao, W., et al. (2018). Novel Electrochemical Sensing Platform for Ultrasensitive Detection of Cardiac Troponin I Based on Aptamer-MoS2 Nanoconjugates. Biosens. Bioelectron. 113, 142-147. doi:10.1016/j.bios.2018.05.003

Regan, B., O'Kennedy, R., and Collins, D. (2018). Point-of-Care Compatibility of Ultra-sensitive Detection Techniques for the Cardiac Biomarker Troponin I-Challenges and Potential Value. Biosensors 8, 114. doi:10.3390/bios8040114 Sarangadharan, I., Regmi, A., Chen, Y.-W., Hsu, C.-P., Chen, P.-c., Chang, W.-H., et al. (2018). High Sensitivity Cardiac Troponin I Detection in Physiological Environment Using AlGaN/GaN High Electron Mobility Transistor (HEMT) Biosensors. Biosens. Bioelectron. 100, 282-289. doi:10.1016/j.bios.2017.09.018

Sun, D., Lin, X., Lu, J., Wei, P., Luo, Z., Lu, X., et al. (2019a). DNA Nanotetrahedron-Assisted Electrochemical Aptasensor for Cardiac Troponin I Detection Based on the Co-catalysis of Hybrid Nanozyme, Natural Enzyme and Artificial DNAzyme. Biosens. Bioelectron. 142, 111578. doi:10.1016/j.bios. 2019.111578

Sun, D., Luo, Z., Lu, J., Zhang, S., Che, T., Chen, Z., et al. (2019b). Electrochemical Dual-Aptamer-Based Biosensor for Nonenzymatic Detection of Cardiac Troponin I by Nanohybrid Electrocatalysts Labeling Combined with DNA Nanotetrahedron Structure. Biosens. Bioelectron. 134, 49-56. doi:10.1016/j.bios. 2019.03.049

Tahernejad-Javazmi, F., Shabani-Nooshabadi, M., and Karimi-Maleh, H. (2019). 3D Reduced Graphene oxide/FeNi3-Ionic Liquid Nanocomposite Modified Sensor; an Electrical Synergic Effect for Development of TertButylhydroquinone and Folic Acid Sensor. Composites B: Eng. 172, 666-670. doi:10.1016/j.compositesb.2019.05.065

Wang, S., Zhao, Y., Wang, M., Li, H., Saqib, M., Ge, C., et al. (2019). Enhancing Luminol Electrochemiluminescence by Combined Use of Cobalt-Based Metal Organic Frameworks and Silver Nanoparticles and its Application in Ultrasensitive Detection of Cardiac Troponin I. Anal. Chem. 91, 3048-3054. doi:10.1021/acs.analchem.8b05443

Wang, Y., Yang, Y., Chen, C., Wang, S., Wang, H., Jing, W., et al. (2020). One-step Digital Immunoassay for Rapid and Sensitive Detection of Cardiac Troponin I. ACS Sens. 5, 1126-1131. doi:10.1021/acssensors.0c00064

Xu, Y., Lu, Y., Zhang, P., Wang, Y., Zheng, Y., Fu, L., et al. (2020). Infrageneric Phylogenetics Investigation of Chimonanthus Based on Electroactive Compound Profiles. Bioelectrochemistry 133, 107455. doi:10.1016/j. bioelechem.2020.107455 
Yan, M., Ye, J., Zhu, Q., Zhu, L., Huang, J., and Yang, X. (2019). Ultrasensitive Immunosensor for Cardiac Troponin I Detection Based on the Electrochemiluminescence of 2D Ru-MOF Nanosheets. Anal. Chem. 91, 10156-10163. doi:10.1021/acs.analchem.9b02169

Ye, J., Zhu, L., Yan, M., Zhu, Q., Lu, Q., Huang, J., et al. (2018). Dualwavelength Ratiometric Electrochemiluminescence Immunosensor for Cardiac Troponin I Detection. Anal. Chem. 91, 1524-1531. doi:10.1021/ acs.analchem.8b04640

Zhang, T., Ma, N., Ali, A., Wei, Q., Wu, D., and Ren, X. (2018). Electrochemical Ultrasensitive Detection of Cardiac Troponin I Using Covalent Organic Frameworks for Signal Amplification. Biosens. Bioelectron. 119, 176-181. doi:10.1016/j.bios.2018.08.020

Zhou, J., Zheng, Y., Zhang, J., Karimi-Maleh, H., Xu, Y., Zhou, Q., et al. (2020). Characterization of the Electrochemical Profiles of Lycoris Seeds for Species Identification and Infrageneric Relationships. Anal. Lett. 53, 2517-2528. doi:10. 1080/00032719.2020.1746327
Zhou, W., Li, K., Wei, Y., Hao, P., Chi, M., Liu, Y., et al. (2018). Ultrasensitive Label-free Optical Microfiber Coupler Biosensor for Detection of Cardiac Troponin I Based on Interference Turning Point Effect. Biosens. Bioelectron. 106, 99-104. doi:10.1016/j.bios.2018.01.061

Conflict of Interest: The authors declare that the research was conducted in the absence of any commercial or financial relationships that could be construed as a potential conflict of interest.

Copyright (c) 2021 Li, Zhang, Zhang, Zhang, Zhang, Zhang, Xuan, Wang, Zhang and Yuan. This is an open-access article distributed under the terms of the Creative Commons Attribution License (CC BY). The use, distribution or reproduction in other forums is permitted, provided the original author(s) and the copyright owner(s) are credited and that the original publication in this journal is cited, in accordance with accepted academic practice. No use, distribution or reproduction is permitted which does not comply with these terms. 